\title{
NUMERICAL OPTION PRICING IN THE PRESENCE OF BUBBLES
}

\author{
ERIK EKSTRÖM ${ }^{1,2}$, PER LÖTSTEDT, LINA VON SYDOW ${ }^{1}$ AND JOHAN TYSK ${ }^{1}$
}

\begin{abstract}
For the standard Black-Scholes equation, there is a unique solution of at most polynomial growth, towards which any reasonable numerical scheme will converge. However, there are financial models for which this uniqueness does not hold, for instance in the case of models for financial bubbles and certain stochastic volatility models. We present a numerical scheme to find the solution corresponding to the option price given by the risk-neutral expectation in the presence of bubbles.
\end{abstract}

\section{INTRODUCTION}

Standard arbitrage theory shows that absence of arbitrage is equivalent to the existence of an equivalent local martingale measure. In most models used for option pricing, including for example the standard Black-Scholes model, the discounted underlying asset is actually a martingale under the pricing measure. However, there are notable exceptions, in which the underlying asset is merely a local martingale. These exceptions include local volatility models in which the volatility function is unbounded at infinity, as well as certain stochastic volatility models with a positive correlation between volatility and stock fluctuations, see [6].

The authors of [1] and [3] propose that a discounted underlying asset which is a strict local martingale (i.e. a local martingale but not a martingale) under the pricing measure can be used to model a financial bubble. In these models several basic properties of option prices may fail such as the put-call parity, the no-dominance principle and the uniqueness of solutions to the corresponding Black-Scholes equation, compare [1], [3] and [4]. For a discussion on existence and uniqueness of solutions to the Black-Scholes equation in one-dimensional diffusion models with bubbles, see [2].

Numerically, it is not clear how to determine the solution corresponding to the risk-neutral expected value. In fact, most numerical methods are set up on a finite grid, and on each such bounded domain, the solution to the Black-Scholes equation is uniquely determined by its boundary conditions. Unless special care is taken when specifying these boundary conditions, the solution will not be the one corresponding to the risk-neutral expected value. In fact, if standard boundary conditions are imposed the desired solution will not be obtained. Thus, for instance, if the boundary condition $x-K e^{-r(T-t)}$ for large values $x$ of the underlying asset is used to determine a call option with strike $K$ at time $T-t$ before maturity, a solution to the Black-Scholes

\footnotetext{
Key words and phrases. Multiple solutions to PDEs; bubbles; option pricing.

${ }^{1}$ Partially supported by the Swedish Research Council (VR).

${ }^{2}$ Partially supported by The Graduate School in Mathematics and Computing (FMB).
} 
equation is indeed obtained but not the solution corresponding to the option price given by a risk-neutral expected value.

In the present paper we provide a method to determine the option price in models with bubbles. The method involves the solution of the Black-Scholes equation on a bounded domain, with a Neumann boundary condition at the spatial boundary. As the state space expands, the solution is shown to converge to the solution given by the risk-neutral expectation. Moreover, the method is easily implemented, and we analyse it numerically.

\section{Model Specification and Numerically Relevant Boundary Conditions}

In this section we study prices of options written on an underlying stock. We model the stock price $X$ under the pricing measure by

$$
d X_{t}=\alpha\left(X_{t}, t\right) d W_{t},
$$

where $W$ is a standard Brownian motion and $\alpha(x, t)$ is a given function. For the sake of convenience we let the interest rate be zero; generalizations to a deterministic interest rate are straightforward. We assume that $\alpha$ is continuous and locally Hölder $(1 / 2)$ in the $x$-variable, and non-zero for all $x>0$. Note, however, that we do not impose any linear bound. We also assume that if $X$ ever reaches zero, then it is absorbed. Financially, this corresponds to the company going bankrupt.

For a given non-negative pay-off function $g$, we define the price at time $t$ of the option that pays $g\left(X_{T}\right)$ at time $T$ to be $u\left(X_{t}, t\right)$, where the function $u$ is given by

$$
u(x, t)=E_{x, t} g\left(X_{T}\right) .
$$

This definition of the option price agrees with the notion of 'fair price' in [1] as the smallest initial endowment needed to form an admissible superreplicating wealth process. Note that $X$ is a lower bounded local martingale, hence a supermartingale. Consequently, $u(x, t)$ is well-defined for any payoff function $g$ of at most linear growth, thus including all standard contracts such as for example call options. In many models, notably the CEV-model considered below, higher moments exist so $u$ would in fact be well-defined for a larger class of pay-off functions. However, since we consider problems for general local volatility models, we will throughout this article consider pay-offs of at most linear growth.

The corresponding Black-Scholes equation is

$$
\left\{\begin{array}{l}
u_{t}(x, t)+\frac{1}{2} \alpha^{2} u_{x x}(x, t)=0 \quad \text { if }(x, t) \in(0, \infty) \times[0, T) \\
u(x, T)=g(x) \\
u(0, t)=g(0) .
\end{array}\right.
$$

It is shown in [2] that if $g$ is of at most linear growth, then the option price $u$ given in (2) is a classical solution to the Black-Scholes equation (3). However, it is well-known that there are multiple solutions of at most linear growth.

Example In the Constant Elasticity of Variance (CEV) model, the stock price is given by

$$
d X_{t}=\sigma X_{t}^{\gamma} d W
$$


where $\gamma \geq 0$ and $\sigma>0$ are constants. If $\gamma>1$, then $X$ is a strict local martingale, compare [1] and [5]. Consequently,

$$
u(x, t)=E_{x, t} X_{T}<x .
$$

On the other hand, $u \equiv x$ is clearly also a solution to the corresponding Black-Scholes equation, so uniqueness of solutions does not hold.

To determine the stochastic solution given by (2), one may employ the following observation made in [2] and [3].

Proposition 2.1. Assume that $g$ is non-negative and of at most linear growth. Then the option price u given by (2) is the smallest non-negative classical solution of (3).

Numerically it is not clear how to check that a given solution to a differential equation is the smallest non-negative one. One possibility to identify this solution would be to replace the pay-off function $g$ by $g \wedge M$ and find the unique bounded solution to the corresponding pricing equation. When letting $M \rightarrow \infty$, the smallest solution is obtained, compare [2]. This method, however, is not straightforward to analyze numerically since, for each $M$, an upper bound for the state space has to be chosen, tending to infinity with $M$. Rather than investigating how to choose the state space bound relative to $M$, we give another characterization of $u$ below which is generally applicable for problems of this type and is also simple to implement.

Let $u_{M}$ be the unique solution to the partial differential equation

$$
\begin{cases}u_{t}^{M}(x, t)+\frac{1}{2} \alpha^{2} u_{x x}^{M}(x, t)=0 & \text { if }(x, t) \in(0, M) \times[0, T) \\ u^{M}(x, T)=g(x) & \text { if } x \in[0, M] \\ u^{M}(0, t)=g(0) & \text { if } t \in[0, T) \\ u_{x}^{M}(M, t)=0 & \text { if } t \in[0, T) .\end{cases}
$$

Note that $u^{M}$ satisfies a Neumann condition at the part of the boundary where $x=M$. We extend the domain of definition of $u^{M}$ by setting $u^{M}(x, t)=u^{M}(M, t)$ for $x \geq M$.

Theorem 2.2. Assume that the pay-off function $g$ is non-negative, nondecreasing and of at most linear growth. Then $u^{M}$ is increasing in $M$, and

$$
u(x, t)=\lim _{M \rightarrow \infty} u^{M}(x, t) .
$$

Proof. Assume that $g$ is non-negative and non-decreasing. To see that $u^{M}$ is increasing in $M$, let $M_{1}$ and $M_{2}$ satisfy $M_{1}<M_{2}$. The monotonicity of $g$ implies that $x \mapsto u^{M_{2}}(x, t)$ is non-decreasing. Consequently, $u^{M_{2}}$ satisfies

$$
\begin{cases}u_{t}^{M_{2}}(x, t)+\frac{1}{2} \alpha^{2} u_{x x}^{M_{2}}(x, t)=0 & \text { if }(x, t) \in\left(0, M_{1}\right) \times[0, T) \\ u^{M_{2}}(x, T)=g(x) & \text { if } x \in\left[0, M_{1}\right] \\ u^{M_{2}}(0, t)=g(0) & \text { if } t \in[0, T) \\ u_{x}^{M_{2}}\left(M_{1}, t\right) \geq 0 & \text { if } t \in[0, T) .\end{cases}
$$

Thus an application of the Maximum principle yields $u^{M_{1}} \leq u^{M_{2}}$. Since $u^{M}$ is increasing in $M$, the limit

$$
\bar{u}(x, t):=\lim _{M \rightarrow \infty} u^{M}(x, t)
$$


clearly exists. Moreover, by a similar argument as above, we have $u^{M} \leq u$ for all $M$, so $\bar{u} \leq u$.

On the other hand, interior Schauder estimates yield that $\bar{u}$ solves the equation (3). Moreover, since $u^{M} \leq \bar{u} \leq u$ for all $M$, and since $u^{M}$ and $u$ are continuous at the boundary, we find that also $\bar{u}$ is continuous at the boundary, with $\bar{u}(x, T)=g(x)$ and $\bar{u}(0, t)=g(0)$. Thus the reverse inequality is a consequence of Proposition 2.1, which proves $\bar{u}=u$.

Corollary 2.3. Assume that the pay-off function $g$ can be written as the difference of two non-negative and non-decreasing functions of at most linear growth. Then

$$
u(x, t)=\lim _{M \rightarrow \infty} u^{M}(x, t) .
$$

Remark Note that Corollary 2.3 covers for example the case of put options. Indeed, $(K-x)^{+}=K-x \wedge K$.

Remark Another possibility to determine the smallest non-negative solution of (3) would be to consider barrier options with a barrier $M$ tending to infinity. This corresponds to solving a partial differential equation with Dirichlet boundary condition at $x=M$. As $M$ tends to infinity, the corresponding solution converges to $u$. However, this method produces an error of size $M$ in supremum norm, so the convergence is slower than in the approximation using a Neumann condition.

\section{Numerical Results}

A numerical solution of (4) is computed on a grid defined by $x_{j}=j h, j=$ $0,1, \ldots, J$, with $x_{J}=M$ and $h=M / J$, and at discrete time points $t^{n}=$ $n \Delta t, n=0,1, \ldots, N$, for a time step $\Delta t=T / N$ by approximating the spatial derivative by centered second-order finite differences. The solution is integrated in time by a $\theta$-method. Then $u_{j}^{n}$ approximating $u^{M}\left(x_{j}, t^{n}\right)$ is determined backward in time starting from $u_{j}^{N}=g\left(x_{j}\right)$ by the recursion

$$
(I-\theta \Delta t A) u^{n}=(I+(1-\theta) \Delta t A) u^{n+1},
$$

where $A$ is the matrix with elements discretizing $\frac{1}{2} \alpha^{2} u_{x x}$.

The boundary condition $u_{x}^{M}(M, t)=0$ in (4) is approximated as $u^{M}(M+$ $h, t)-u^{M}(M, t)=0$ and consequently $u_{J}^{n}=u_{J+1}^{n}$. One can of course consider alternatives for the numerical boundary condition. The disadvantage of a Dirichlet condition is that it is unclear what boundary values to impose (see the discussion in the Introduction). A numerical condition such as extrapolation by letting the second difference of $u^{n}$ at $x_{J}$ be zero leaves the initial $u_{j}^{N}=x_{j}$ unaltered and converges to the wrong solution (cf. the Example in Sect 2.).

Following the arguments in [7, Ch. III.10], one can show that the discretization in (5) and the simplest extrapolation boundary condition is stable in a discrete weighted $l_{2}$-norm for all $\Delta t$ if $\theta$ satisifes $1 / 2 \leq \theta \leq 1$ and with time step restrictions if $0 \leq \theta<1 / 2$. The accuracy in $u_{j}^{n}$ is of $\mathcal{O}\left(\Delta t+h^{2}\right)$ if $\theta \neq 1 / 2$ but for $\theta=1 / 2$ we have the Crank-Nicolson method and an accuracy of $\mathcal{O}\left(\Delta t^{2}+h^{2}\right)$. The method is also stable in the maximum norm if $(1-\theta) \Delta t \alpha\left(x_{j}\right)^{2} / h^{2} \leq 1$ for all $j$, see [7, Ch. III.11]. Furthermore, under 
the same condition the solution satisfies a discrete maximum principle: $u_{j}^{n}$ has its maximum initially at $n=N$ or at a boundary $j=0$ or $j=J[7$, Ch. III.11].

Stability and consistency imply that the computed solution $u_{j}^{n}$ converges to $u^{M}\left(x_{j}, t^{n}\right)$ when $\Delta t \rightarrow 0$ and $h \rightarrow 0$. Then $u^{M}$ converges to $u$ as $M \rightarrow \infty$ according to Theorem 2.2. Hence, the solution of (5) converges to $u$ when the solution interval is expanded.

Two model problems are solved, both using the Constant Elasticity of Variance model with $\gamma=2$ and $\sigma=0.1$. The first case in Figure 1 is the stock option with $g(x)=x$, and the second case is a European call option with strike price $K=5$ in Figure 2. In this case $g(x)=\max (0, x-K)$. The parameters in our experiments are $\theta=1$ (the implicit Euler method), $h=0.05, \Delta t=0.0005$, and $T=0.5$. We choose $h$ and $\Delta t$ such that the error compared to $u$ due to them is much smaller than the error caused by a finite $M$.

The solution is computed for $M=2^{m} \cdot 10, m=1,2,3,4,5,6$. A close-up view of the solutions is depicted in the left panels of the figures. Only the parts of the solutions are displayed where a different $M$ has an influence. The solutions with $M=320$ and $M=640$ are on top of each other for $x \leq 320$. The solution for a fixed $x$ increases as $M$ increases in agreement with the proof of Theorem 2.2. The differences between the analytical solutions in [1] and numerical solutions obtained by (5) are measured at $t=0$ by $\delta$ defined by

$$
\delta^{2}=\frac{1}{M} \sum_{j=1}^{J} h\left|u\left(x_{j}, 0\right)-u_{j}^{0}\right|^{2}=\frac{1}{J} \sum_{j=1}^{J}\left|u\left(x_{j}, 0\right)-u_{j}^{0}\right|^{2}
$$

in the right panels of the figures. The slopes in Figures 1 and 2 suggest that the difference decays as $M^{-2}$.

\section{REFERENCES}

[1] Cox, A. and Hobson, D. Local martingales, bubbles and option prices. Finance Stoch. 9 (2005), 477-492.

[2] Ekström, E. and Tysk, J. Bubbles, convexity and the Black-Scholes equation. Ann. Appl. Probab. 19 (2009), 1369-1384.

[3] Heston, S., Lowenstein, M. and Willard, G. Options and bubbles. Rev. Financ. Stud. 20 (2007), 359-390.

[4] Jarrow, R., Protter, P. and Shimbo, K. Asset price bubbles in complete markets. In Advances in mathematical finance, Appl. Numer. Harmon. Anal., pages 97-121. Birkhäuser Boston, Boston, MA, 2007.

[5] Lewis, A. L. Option valuation under stochastic volatility. With Mathematica code. Finance Press, Newport Beach, CA, 2000.

[6] Sin, C. Complications with stochastic volatility models. Adv. Appl. Probab. 30 (1998), 256-268.

[7] Thomée, V. Finite difference methods for linear parabolic equations, in Handbook of Numerical Analysis, eds. P. G. Ciarlet, J. L. Lions, North Holland, Amsterdam, 1990, 5-196.

E-mail address: ekstrom@math.uu.se, perl@it.uu.se, lina@it.uu.se, Johan.Tysk@math.uu.se 


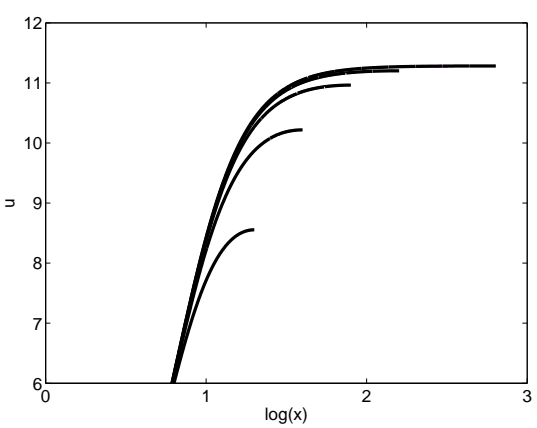

(a)

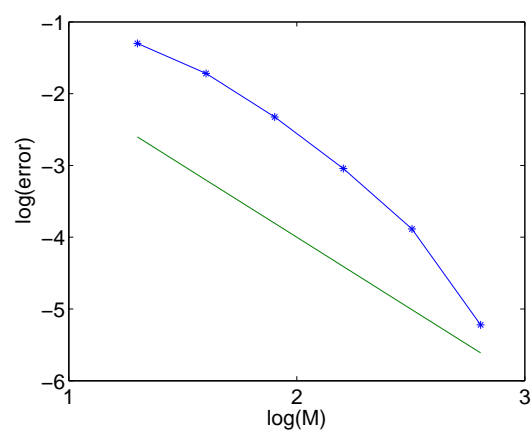

(b)

Figure 1. (a) Close-up view of the solution of the stock option example for different $M$. (b) The difference between the analytical solution and the numerical solution for different $M\left(^{*}\right)$. The linear curve indicates a decay $\sim M^{-2}$.

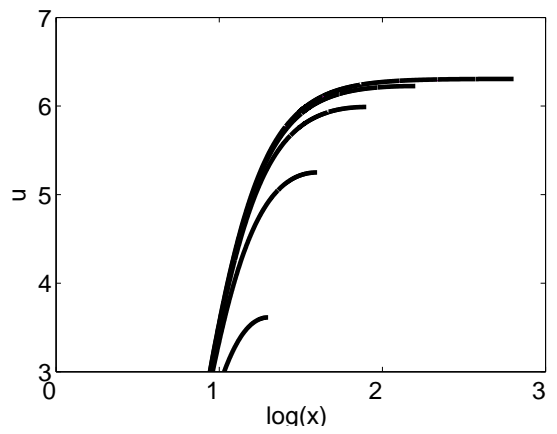

(a)

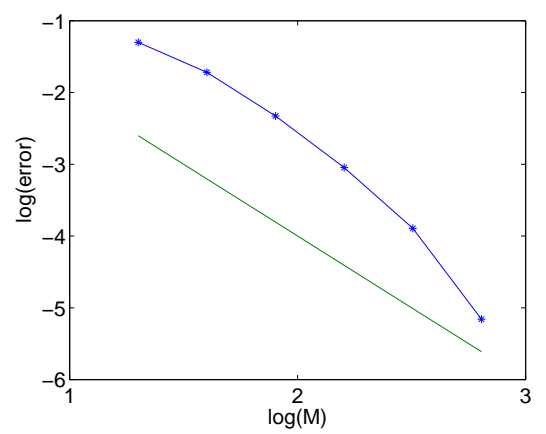

(b)

Figure 2. (a) Close-up view of the solution of the call option example for different $M$. (b) The difference between the analytical solution and the numerical solution for different $M\left({ }^{*}\right)$. The linear curve indicates a decay $\sim M^{-2}$. 